\title{
Determinants of the Perception of Rotational Motion: Orientation of the Motion to the Object and to the Environment
}

\author{
John R. Pani, Colin T. William, and Gordon T. Shippey \\ Emory University
}

\begin{abstract}
The results of two experiments suggest that strong constraints on the ability to imagine rotations extend to the perception of rotations. Participants viewed stereographic perspective views of rotating squares, regular polyhedra, and a variety of polyhedral generalized cones, and attempted to indicate the orientation of the axis and planes of rotation in terms of one of the 13 canonical directions in $3 \mathrm{D}$ space. When the axis and planes of a rotation were aligned with principal directions of the environment, participants could indicate the orientation of the motion well. When a rotation was oblique to the environment, the orientation of the object to the motion made a very large difference to performance. Participants were fast and accurate when the object was a generalized cone about the axis of rotation or was elongated along the axis. Variation of the amount of rotation and reflection symmetry of the object about the axis of rotation was not powerful.
\end{abstract}

The study of motion and spatial transformation has long been central in mathematics and the physical sciences, and recently it has become the focus of much work in the study of perception and spatial cognition. Rotation, for example, is a fundamental form of motion (e.g., Gibson, 1957; Shepard, 1984), and the study of mental imagery has benefited greatly from the investigation of mental imagery of rotation (see Shepard \& Cooper, 1982). Across the study of spatial cognition, it has become clear that some forms of spatiotemporal structure are cognitively simple for the typical person, whereas other forms are quite complex and difficult. This distinction is familiar from work on the spatial organization of elementary forms (e.g., Garner, 1974; Palmer, 1977; Wertheimer, 1950), but it applies also to a great variety of familiar or three-dimensional (3D) structures and events (e.g., Hinton, 1979; McCloskey, 1983; Pani, 1993; Pani, Zhou, \& Friend, 1995; Proffitt, Kaiser, \& Whelan, 1990; Tversky, 1981).

Consider simple rotational motion, the topic of this article. In simple rotation, all of the points on a rotating object move, with common angular velocity, in circles about an axis fixed in space. The planes of these circles are parallel to each other and normal to the axis (e.g., Todd, 1982). In contrast, if the axis and planes of rotation change orientation during the motion, the rotation is no longer physically simple. Fundamental parameters of simple rotation include the orientation of the axis and planes of rotation to the environment and the orientation of the rotating object to the axis and planes of rotation (see Pani, 1989, 1993; Pani \&

John R. Pani, Colin T. William, and Gordon T. Shippey, Department of Psychology, Emory University.

We thank Carolyn Mervis, Stephen Palmer, and Margaret Shiffrar for helpful comments on earlier versions of this article.

Correspondence concerning this article should be addressed to John R. Pani, Department of Psychology, Emory University, Atlanta, Georgia 30322. Electronic mail may be sent via Internet to pani@fs1.psy.emory.edu.
Dupree, 1994; Shiffrar \& Shepard, 1991). In Figure 1, for example, the rods are axes of rotation with fixed directions in space; when the rods spin, the squares rotate about the rods. In the system in Figure 1A, the rod is aligned with the environmental vertical and the square is aligned with the rod. If participants are asked to indicate what the orientation of the square would be after a rotation of the rod, say $180^{\circ}$, they succeed easily. In Figure 1B, the rod is aligned with the environment, but the square is oblique to the rod. In Figure $1 \mathrm{C}$, the rod is oblique to the environment, but the square is aligned with the rod. In both of these mixed cases, participants can imagine the rotations rather well. The system in Figure 1D is double oblique and is impossible for the typical participant to imagine, even when the mean response time is over 2 min (Pani, 1993; Pani \& Dupree, 1994; see also Just \& Carpenter, 1985; Massironi \& Luccio, 1989; Parsons, 1987; Shiffrar \& Shepard, 1991). As most readers are unable to predict the outcome of the rotation suggested in Figure $1 \mathrm{D}$, three orientations from a $180^{\circ}$ rotation of this system are illustrated in Figure 2.

A more succinct summary of these findings is that people are able to predict the outcome of a rotation only if the axis and planes of rotational motion are aligned with a salient spatial reference system, generally the principal directions of the environment or the intrinsic reference system of the object (Massironi \& Luccio, 1989; Pani, 1993; Pani \& Dupree, 1994; Shiffrar \& Shepard, 1991). In the rotation of Figure 1D, the axis and planes of rotation are aligned with neither of these reference systems, and performance is markedly poor. Why should alignment of a rotational motion with a salient reference system be so important to imagination of the motion?

We suggest that for the typical person, organization of rotational motion includes two components. Most generally, the person must be able to organize the circular motions that are contained within the planes of rotation (Pani, 1993; Pani \& Dupree, 1994; Todd, 1982). If an object has no definite 
Orientation of the Object to the Axis of Rotation

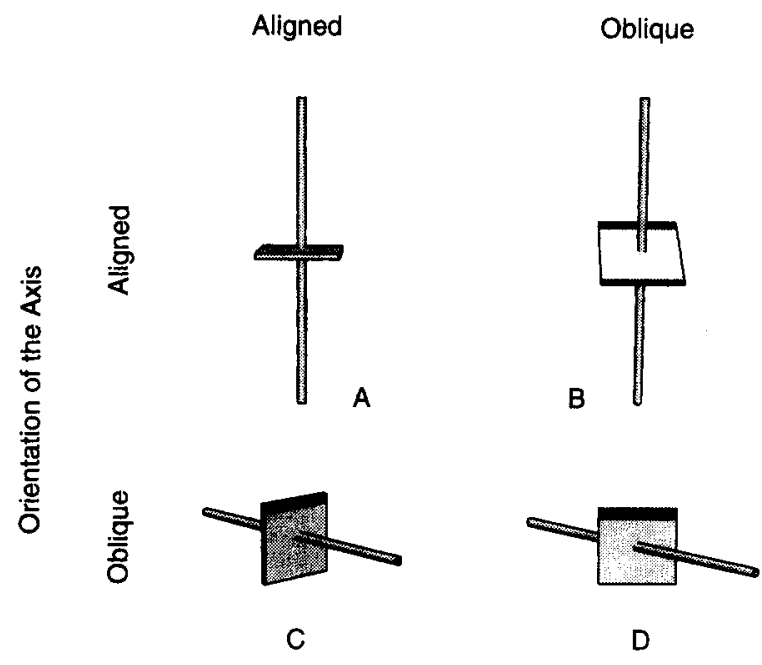

Figure 1. If the rods are axes of rotation with a fixed direction, these four assemblies illustrate basic types of rotational motion.

orientation (e.g., if it can be seen as an isotropic cloud of dots), then there is little difficulty in imagining these circular motions of the object about an arbitrary axis in space. However, if an object has a definite orientation, as a square does, then there is an additional component in the organization of rotation: The person must be able to see the object as having invariant slant to the axis of rotation. Only then can the individual imagine the circular motion of the object about the axis. For example, to correctly imagine the rotation in Figure 1D, the individual must be able to perceptually organize the physical assembly in terms of the invariant $45^{\circ}$ angle of the square to the rod. Then this square-at $-45^{\circ}$ can be imagined to spin around the rod.

Axes of rotation are weak reference axes for the typical person, and to maintain the axis-relative description of the slant of the square, the individual requires reinforcement of the axis from another reference system. Thus, if the rod and square assembly is placed so that the rod is aligned with the vertical, as in Figure 1B, the invariant $45^{\circ}$ slant of the square to the axis of rotation is easily understood; the square has the same angle to the vertical, and the vertical is a strong reference axis. Alternatively, if the square is made perpendicular to the rod, as in Figure 1C, the invariant slant of the square to the axis again is obvious. The axis of rotation is aligned with a direction that is recognized to be part of an object-relative reference system (in this instance, a surface normal).

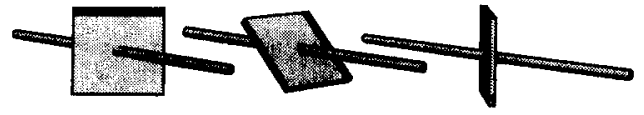

Figure 2. Three orientations of the square in a double-oblique rotation.
Note that this view stresses the importance of alignment, the presence of orientations that are parallel or perpendicular to a relevant spatial reference system. The canonical nature of aligned orientations has been demonstrated in a variety of tasks. For example, people are more sensitive to deviation from parallelism than from arbitrary angles (Goldmeier, 1972; Rock, 1983). An oblique line "pops out" more readily in a field of vertical lines than does a vertical line in a field of obliques (Treisman \& Gormican, 1988). People are most sensitive to bilateral symmetry about a vertical axis and least sensitive to symmetry about an oblique axis (Palmer \& Hemenway, 1978). (Pani, Jeffres, Shippey, \& Schwartz, in press, provide a more extensive discussion of alignment; see also Appelle, 1972; Howard, 1982; Olson, 1970; Shiffrar \& Shepard, 1991.)

A primary goal of this project was to learn whether constraints on the imagination of rotational motion also are strong in the perception of rotation (see also Kaiser, Proffitt, \& Anderson, 1985; Proffitt \& Gilden, 1989). That is, if a simple rotation is particularly difficult for people to imagine, and a person has an opportunity to view and study such a rotation, will it be especially difficult for the person to see that the displayed motion is a simple rotation? Given the findings concerning the imagination of rotation, predictions for the perception of rotation would seem to be fairly straightforward. First, if the axis of a simple rotation is aligned with the environment, particularly the vertical, the person will readily perceive the motion to be a simple rotation, independent of the orientation of the rotating object to the axis of rotation. Second, oblique-axis rotations only will be perceived to be simple rotations if the rotating objects are aligned with the axes of rotation. The question arises, however, as to what properties of objects are critical in this context. There is common ground here for theories of motion perception and theories of form perception and object recognition.

Object recognition implies, among other things, perceived invariance of the structural properties of an object across changes of position and orientation. Theorists account for this invariance by positing an object-relative reference system to which cardinal directions (e.g., top and bottom) and spatial descriptors (e.g., flat or pointed) can be related (Biederman \& Gerhardstein, 1993; Hinton, 1979; Marr \& Nishihara, 1978; Palmer, 1975, 1989; Rock, 1983). The well-known demonstrations of change of form perception with reorientation of an object are instances in which there is not a singular object-relative reference system that provides such invariance (Koffka, 1935; Mach, 1906/1959; Rock, 1973; see Palmer, 1989). It is sensible to suppose, then, that if a rotational motion is oblique to the environment, the rotation will appear simple when the objectrelative reference system of the rotating object is aligned with the motion. Clear candidates for object structures that would determine at least the major axis of an object-relative reference system are the generalized cone (Biederman, 1987, 1990; Binford, 1971; Marr \& Nishihara, 1978); elongation of the object (Humphreys, 1983; Marr \& Nishihara, 1978; Palmer, 1989); and rotation and reflection symmetry (Garner, 1974; Palmer, 1985, 1989; Palmer \& Hemenway, 
1978; Pomerantz \& Kubovy, 1986; Shiffrar \& Shepard, 1991). A generalized cone is any shape that could be "swept out" by moving a cross-section of constant shape along a line, where the cross-section could change in size (Binford, 1971).

The present experiments were designed to investigate the effects of the orientations of rotational motions to the environment, and the orientations of the rotating objects to the motions, on the perception of the rotations. If the environment is considered to contain a Cartesian reference system (e.g., the 6 surfaces of a standard room and the edges formed by their intersection), the axis of rotation either was aligned with a principal axis of the environment (i.e., vertical or frontal-horizontal), partially oblique to the environment (i.e., contained within the horizontal plane but at $45^{\circ}$ to the axes in that plane), or fully oblique to the environment (i.e., oblique to all principal axes and planes). Object structures that could be aligned with the rotation included the generalized cone, elongation, and rotation and reflection symmetry. The objects used in these experiments were primarily the square and the three simplest Platonic solids, as illustrated in Figure 3. The Platonic solids are the five regular polyhedra, including the tetrahedron, cube, octahedron, dodecahedron, and icosahedron. They are treated as fundamental forms in every intellectual discipline that describes spatial structure (e.g., Gasson, 1983; Hilbert \& Cohn-Vossen, 1952; Holden, 1971; Kappraff, 1991). Of particular relevance here is that the tetrahedron, cube, and octahedron, as well as the square, are relatively simple; they are generalized cones (Pani \& Zhou, 1993; Pani, Zhou, \& Friend, 1995); and they have different amounts of symmetry when placed in various orientations to an axis (e.g., Holden, 1971; Kappraff, 1991). As with any object, it also is possible to elongate them along a given axis.

The displays in these experiments were computer simulations of opaque rotating objects. Displays were stereographic and rendered in perspective. With the added benefit to depth perception of apparent rotation in depth (Lappin, Doner, \& Kottas, 1980; Todd, 1982; Ullman, 1979; Wallach \& O'Connell, 1953), there was vivid perception of rigid objects rotating in $3 \mathrm{D}$ space. In selecting an experimental task, considerations of the kinematics of rotation suggested that accuracy and response time in indicating the orientation of the axis and planes of rotation would be relatively direct measures of whether the participant perceived a simple rotational motion. Participants used a rod and attached disc for this purpose. There was little demand for precision; the orientations of the rotations and the response alternatives were limited to the canonical orientations that occur in
Square
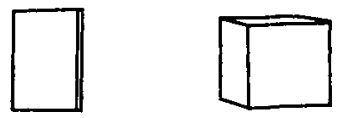

Octahedron

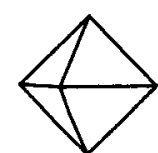

Tetrahedron

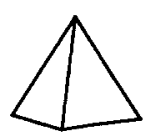

Figure 3. The objects shown rotating in Experiment 1. $35-45^{\circ}$ intervals. It was intended that with these large intervals, failures to correctly indicate the orientations of particular rotations, especially if accompanied by long response times, would imply an inability to perceive these motions as simple rotations.

Two studies of the perception of rotation are particularly relevant to the present experiments. First, Hecht and Proffitt (1991) found that participants generally were able to perceive double-oblique rotations correctly in apparent motion displays. An important difference between their experiments and our own is that the axis of rotation in the Hecht and Proffitt study was not through the object. In that case, the motion can be perceived as a curved path within a plane, much like runners moving about a track. It may be possible for people to see this curved path as defining a plane of motion, with the object having a constant oblique angle with respect to that plane. The slanted blades of propellers probably are conceptualized in this way. We think this difference in results points to a distinction between two psychologically different types of motion (see also Cutting \& Proffitt, 1982).

Shiffrar and Shepard (1991) examined the recognition of rotations of a cube in which the axis of rotation went through one-, three-, or fourfold symmetry axes of the cube and was at different orientations with respect to the environment. Recognition was better at higher symmetries and when the rotation was aligned with the environment and viewer. The present experiments differ from their study in two important ways. First, the present experiments displayed the complete set of rotational symmetries for four objects rather than one. The comparison across objects permits disentangling a number of variables that covary with symmetry within a single object. When the cube is maximally aligned with an axis of rotation-for example, it has a very high degree of symmetry about the axis--it is a generalized cone about the axis, and all its edges and surfaces are aligned with the axis. Second, the tasks are quite different. Shiffrar and Shepard used a same-different recognition task, whereas the task in the present experiments was to describe single rotations. It was expected that the descriptive task would replicate the extreme range of performance found in studies of the imagination of rotation (Pani, 1993; Pani \& Dupree, 1994).

\section{Experiment 1}

In Experiment 1, participants viewed rotations of the square and the three simpler Platonic solids. The Platonic solids are described in standard geometric treatments in terms of their rotation and reflection symmetries relative to axes of the objects (e.g., Hilbert \& Cohn-Vossen, 1952; Holden, 1971; Kappraff, 1991). In particular, the cube and octahedron have axes of fourfold, threefold, and twofold symmetry, and the tetrahedron has axes of threefold and twofold symmetry, as illustrated in Figure 4. For example, a line running through the middle of opposite faces of the cube is an axis of fourfold rotational symmetry. If the cube is rotated about this axis, it occupies exactly the same space 


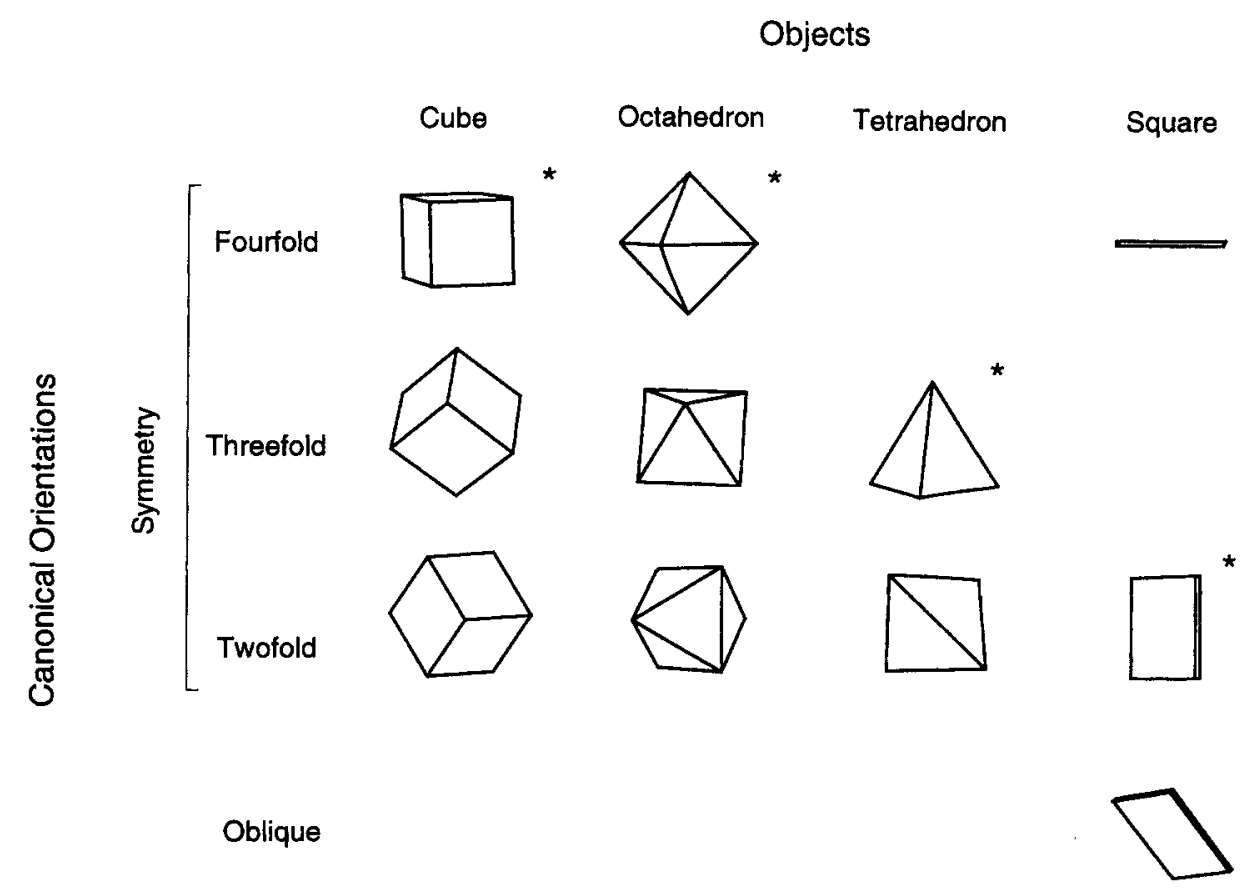

Figure 4. Canonical orientations of the objects in Experiment 1, relative to the vertical. The orientations with asterisks indicate that the object is a generalized cone at this orientation to the vertical.

four times during one revolution. For each of the Platonic solids, there are sets of planes of reflection symmetry that correspond to the axes of rotational symmetry. For example, the cube has four planes of reflection symmetry coincident with its axis of fourfold rotational symmetry. The square also has different levels of symmetry as it is oriented through canonical angles relative to an axis. Most important, the square has fourfold symmetry when it is normal to the axis and twofold symmetry when it is parallel to the axis. The square has one additional canonical orientation: oblique to an axis at $45^{\circ}$. The orientations that result from aligning the symmetry axes of these four objects to a reference axis, and the oblique orientation of the square, will be referred to as the canonical orientations of the objects. In this experiment, each object was placed in all its canonical orientations to the axis of rotation. If the amount of symmetry of an object aligned with the axis and planes of rotation was of fundamental importance to the perception of rotation, it should have been easier to detect simple rotational motion with higher levels of symmetry across the four objects.

The square and the three simpler Platonic solids also are generalized cones (Pani \& Zhou, 1993; Pani, Zhou, \& Friend, 1995). As noted earlier, a generalized cone is any shape that could be formed by moving a cross-section of constant shape along a line where the cross-section could change in size (Binford, 1971). Prominent theories of object recognition have suggested that the generalized cone is a volumetric primitive in the representation of the form of objects (Biederman, 1987, 1990; Brooks, 1981; Marr, 1982;
Marr \& Nishihara, 1978). The cube and octahedron are generalized cones about their fourfold axes of symmetry, the tetrahedron is a generalized cone about its threefold axis of symmetry, and the square is a generalized cone about its twofold axis of symmetry. The square also is a minimal generalized cone, a single cross-section, about its fourfold axis. Note that these objects are generalized cones of a particular type. They have straight axes and cross-sections that are normal to the axes. If the structure of this rather "standard" generalized cone is salient in the perception of rotations, rotation about the conic axes should always appear simple even though the objects are generalized cones at different levels of symmetry.

It is important for the investigation of global properties of these objects that the cube and octahedron are geometric duals of each other (Hilbert \& Cohn-Vossen, 1952; Holden, 1971). That is, these two objects both are generalized cones and have the same symmetries, but everywhere that one object has a corner, the other object has a surface, and vice versa. Thus, the global properties of these objects can be studied independently of local properties such as the orientation of individual edges and surfaces.

\section{Method}

Participants. Participants were 18 Emory University undergraduates (10 women and $8 \mathrm{men}$ ) in an introductory course in psychology who received course credit for their involvement in the study.

Materials. Rotating cubes, octahedra, tetrahedra, and squares 
were displayed stereographically as central projections of opaque objects. The displays were presented on a computer monitor of 896 by 672 pixels at a resolution of $72 \mathrm{dpi}$. The stereographic aspect of the displays consisted of the presentation of two views of an object, one for each eye, approximately centered within the lateral halves of the monitor (i.e., with a distance of $15 \mathrm{~cm}$ between the centers of the two views). Participants viewed the displays through a set of four front-surface mirrors, as illustrated in Figure 5. The images were accurate in size, linear perspective, and stereo disparity for objects of given sizes located $20 \mathrm{~cm}$ behind the screen and viewed from a distance of $1 \mathrm{~m}$. The sizes of the virtual objects were an average of $4.9 \mathrm{~cm}$ on an edge. The objects were displayed with black edges that were 3 pixels wide and were on white surfaces. An edge of average length would subtend $2.8^{\circ}$ of visual angle when in the plane of the screen.

The rotating objects were shown within a white circular field surrounded by a black outer region, as illustrated in Figure 6 . The circular fields were $11.3 \mathrm{~cm}$ in diameter and were set at a stereo disparity such that the black outer region appeared in the frontal plane at the location of the screen and the objects appeared to be viewed through a round aperture, like a porthole. The white circular field appeared as the visible portion of an open illuminated area behind the porthole. The room was darkened, and no other context was visible.

The mirrors were held securely by a stand $58 \mathrm{~cm}$ from the monitor, at the height of the display images. There was a small screen between the viewer and the mirrors with a $2.9-\mathrm{cm}$ square aperture in front of each inner mirror. Participants sat in a chair and looked into the inner mirrors as if through a pair of binoculars fixed in space. The only view was of the portion of the monitor that held the display images. The outer mirrors were at $45^{\circ}$ to the monitor and separated by the same distance as the two display images $(15 \mathrm{~cm})$. The inner mirrors were at $44.2^{\circ}$ to the cyclopean line of sight of the participant. This slight deviation from $45^{\circ}$ meant that a person with an interocular distance of $5.7 \mathrm{~cm}$ stationed $15.2 \mathrm{~cm}$ from the mirrors would fuse the images with ocular convergence appropriate for looking at a point on the monitor. In addition, people with different interocular distances could move slightly forward or backward to have comfortable convergence and fusion. Thus, it was not necessary to change the distance between the mirrors for each participant. As noted earlier, the images on the screen implied the presence of objects that were $20 \mathrm{~cm}$ behind the screen, for an apparent viewing distance of $1 \mathrm{~m}$.

Each object was displayed in every canonical orientation to the axis of rotation (see Figure 4). There were three categories of orientation of the axis of rotation to the environment: aligned, partially oblique, and fully oblique. There were two instances of each of these categories, as illustrated in Figure 7 . Each of these

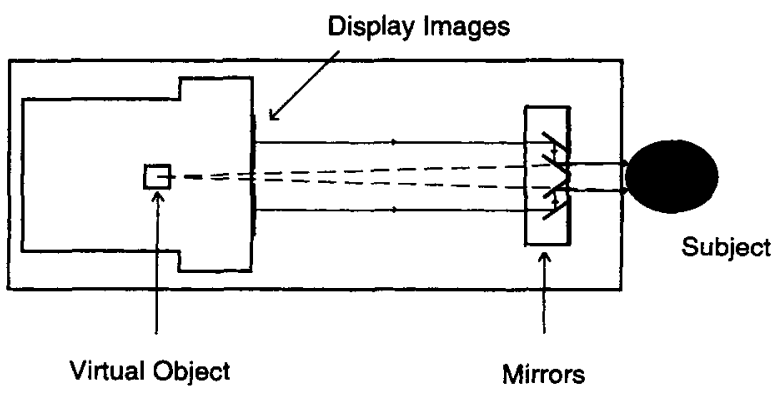

Figure 5. Diagram of the viewing apparatus used in Experiments 1 and 2 .

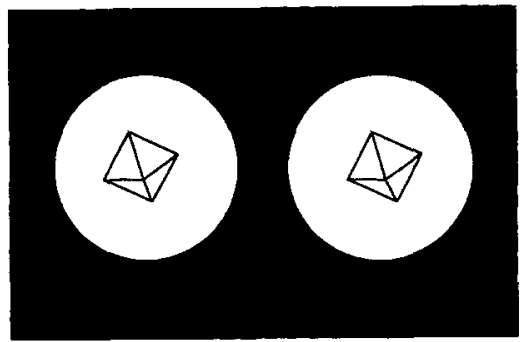

Figure 6. Example of one frame (reduced in size) from an experimental display.

six orientations of the axis of rotation could have a rotation in either direction about the axis, making 12 axis-direction combinations in all. The complete display set included rotations of each of the four objects, in each canonical orientation to the axis of rotation, for each of the axis-direction combinations. The rotations were shown at 120 frames per revolution (with a $3^{\circ}$ change of orientation between each frame), at a speed of one revolution per $6 \mathrm{~s}$.

A set of precise models of each orientation of each object to the axis of rotation was used in the instructions. The models averaged $5.6 \mathrm{~cm}$ on an edge, were made of heavy white cardboard, and had clearly delineated edges (i.e., the surfaces were joined at the inside edges so that the thickness of the adjoining surfaces formed a gray channel between the surfaces). Each model had a dowel running through the center of the object, coincident with one of the object axes. The dowels were $1 \mathrm{~cm}$ in diameter, $60 \mathrm{~cm}$ in length, and were painted flat white. In addition, there was a model of each object without a dowel.

The participant's task was to indicate the orientation of the axis and planes of an ongoing rotation. The response apparatus is illustrated in Figure 8. The participant used a thin dowel, $0.5 \mathrm{~cm}$ in diameter and $25 \mathrm{~cm}$ in length, that was perpendicular through the center of a white foamboard disc $12.5 \mathrm{~cm}$ in diameter. The disc had a semicircular double-headed arrow on one side to indicate that the disc represented the circular motion of the rotation. To indicate the orientation of a rotation, the participant first held the disc and dowel in the desired orientation and then inserted the dowel into a white polyhedron, $6.5 \mathrm{~cm}$ across, that had holes through it in all 13 principal directions of 3D space, in $35-45^{\circ}$ intervals. The surfaces of the polyhedron faced in the same directions as the holes. Once the dowel was put through a hole, the disc rested against the polyhedron, and the dowel and disc were held at a given orientation. The polyhedron was mounted on a gray stand at the height of

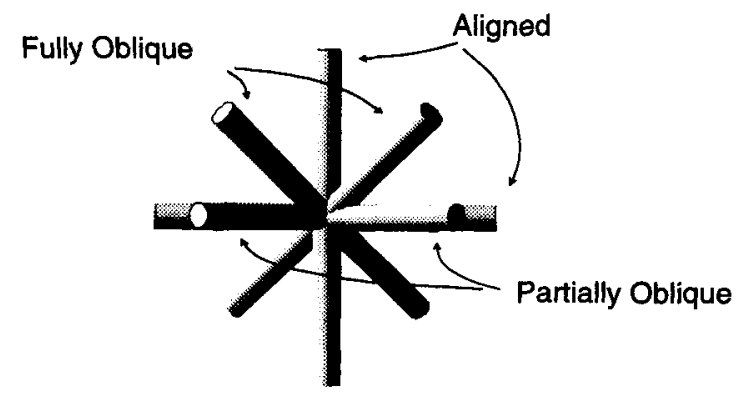

Figure 7. The directions of the axes of rotation used in Experiment 1 . 


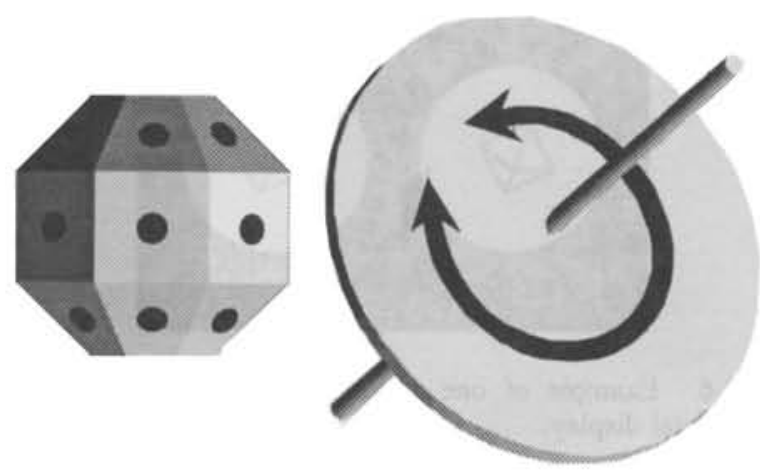

Figure 8. The response apparatus in Experiments 1 and 2.

the rotating shapes, and was positioned in front of the participant and off to the left side, just out of the line of sight to the display monitor. The polyhedron was attached to the stand by a pipe that extended horizontally out the back of the polyhedron.

Procedure. The first step of the procedure was a demonstration of the stereo viewing apparatus. The participant next read a set of well-illustrated written instructions. The instructions explained that (a) rotations produce circular paths of the points on an object in parallel planes of rotation; (b) there is a line, called the axis of rotation, that runs through the middle of the planes of rotation; (c) the axis and planes of rotation can be oriented in any direction in space; (d) an object need not be aligned with the axis and planes of rotation in any particular way; and (e) the participant's task would be to view a rotating object and to identify the orientation of the axis and planes of rotation.

After the written instructions, the experimenter reviewed these main points using squares attached to rods. Each rod was held frontal-horizontal relative to the participant, and rotations were demonstrated-first with the square normal to the rod, then parallel to the rod, and finally oblique to the rod. It was emphasized that so long as the rod was in one orientation, the axis and planes of motion were the same in the three cases and that the same answer should be given for them.

The next phase of instruction was to demonstrate the response apparatus. The participant was told that the disc represented the planes of rotation, whereas the stick represented the axis. There were then at least six practice trials in which the participant reproduced a systematic variety of orientations of a second disc and stick held by the experimenter in front of the computer monitor. Practice in indicating orientations continued until the participant could accurately reproduce each orientation on the first attempt. Participants appeared to find the response system intuitive. During the experiment, it was common for participants to insert the stick into the polyhedron and then to spin the assembly in the correct direction to fully represent the rotation. The next phase of instruction was two practice trials with the procedure to be used in the experimental trials. The practice trials displayed an object that was not seen later in the experiment (an antiprism with a square top and bottom; Holden, 1971) rotating about an oblique axis of rotation. One trial showed the object aligned with the axis of rotation, and one trial showed the object oblique to the axis. The participant was informed that viewing time would be recorded but that being as accurate as possible was the important consideration.

Trials were blocked by object, with the participant taking a rest between blocks. Before each block, the cardboard model of the object was given to the participant to explore visually and manually. At the same time, the experimenter took each model of the object that corresponded to a separate canonical orientation. The experimenter held up the model, pointed out how the rod ran through the object (e.g., through opposite corners of the object or through opposite faces), and then suggested that the object might rotate about such an axis. He then showed the rod at five different orientations in $3 \mathrm{D}$ space. At the end of the demonstration for that object, the participant was reminded that the task was to demonstrate the orientation of the axis and planes of rotation.

Individual trials proceeded according to the following sequence. At the beginning of the trial, the circular surround showed a fixation dot at the center. The participant pressed the spacebar on the computer keyboard to initiate the display and then attempted to decide the orientation of the rotation without handling the orientation indicator (i.e., the stick and disc). When the participant knew the answer, or when he or she thought that no further progress could be made, the participant picked up the orientation indicator. At that time, the participant was free to use the indicator to help decide on a response. When the participant had his or her answer, the stick was placed in the polyhedron in the appropriate hole. The experimenter pressed the mouse key once when the response disc was picked up and a second time when the participant placed the indicator in the polyhedron and stated that he or she was finished (typically by saying "OK"). The display remained on the screen and the participant was permitted to view it until he or she was finished (i.e., the second mouse click). The duration of viewing was recorded by the computer at both mouse clicks. The orientation of the indicator was recorded by the experimenter with a simple analog coding system.

Every participant saw all four objects in all combinations of the two basic variables: the three categories of orientation of the axis of rotation and the canonical orientations of the objects to the axis of rotation. Thus, each participant had nine trials with the cube, nine with the octahedron, six with the tetrahedron, and nine with the square. There were four separate lists of rotations for each object. In each list, the rotations were sampled from the larger set of available rotations (described earlier). There also were eight different trial orders. In each, the order of rotations was random with the constraint that a particular orientation of the axis of rotation and a particular orientation of the object to the axis were not repeated on successive trials. The order of objects and the association of objects with lists and trial orders were counterbalanced across participants according to Latin squares.

\section{Results}

Accuracy. The percentage of responses that were correct, as a function of object, orientation of the axis of rotation to the environment, and orientation of the object to the axis of rotation, is presented in Figure 9. For each of the objects, the orientation of the axis of rotation had a large effect on performance: cube, $F(2,32)=9.34, p<.001$; octahedron, $F(2,32)=20.07, p<.001$; tetrahedron, $F(2$, $32)=7.65, p<.01$; square, $F(2,32)=10.38, p<.001$. Planned comparisons between pairs of orientations revealed statistically reliable differences between the aligned orientations and both the partially and fully oblique orientations, respectively: cube, $F(1,16)=8.49, p<.01 ; F(1,16)=$ $14.40, p<.01$; octahedron, $F(1,16)=30.65, p<.001$; $F(1,16)=26.27, p<.001$; tetrahedron: $F(1,16)=22.88$, $p<.001 ; F(1,16)=9.33, p<.01 ;$ square, $F(1,16)=$ $19.32, p<.001, F(1,16)=15.81, p=.001$. Differences between the two oblique orientations were not statistically reliable. 


\section{Object Axis Aligned with the Axis of Rotation}

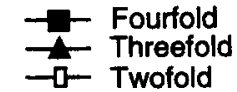

Cube

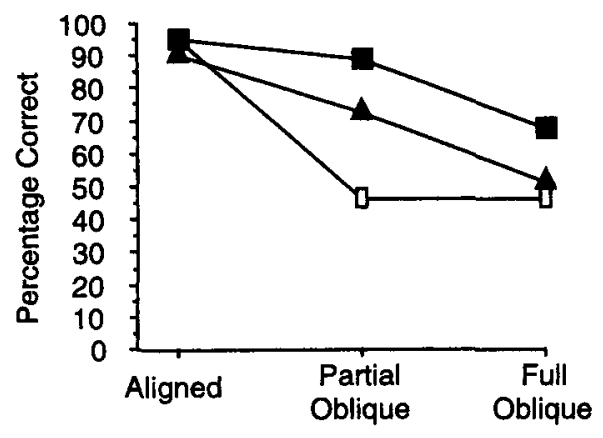

Tetrahedron

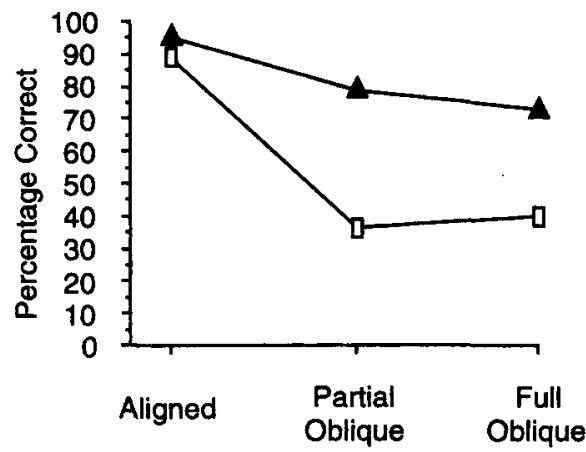

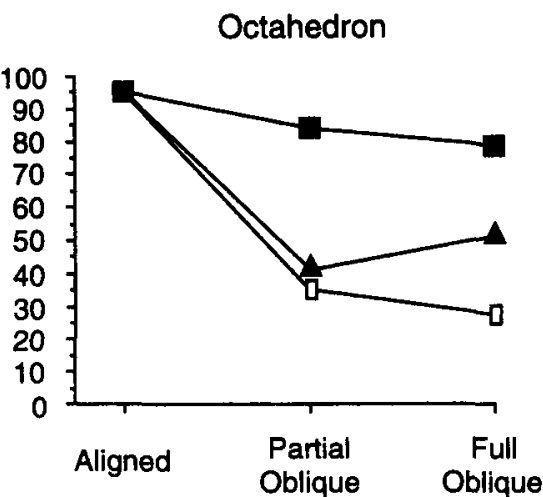

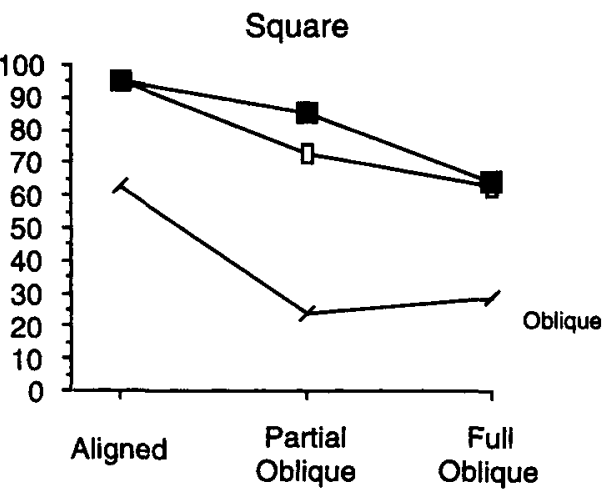

\section{Orientation of the Axis of Rotation}

Figure 9. Percentage correct in Experiment 1 as a function of object, orientation of the axis of rotation, and orientation of the object to the axis of rotation.

For each of the objects, the orientation of the object to the axis of rotation had a large effect on performance: cube, $F(2,32)=5.47, p<.01$; octahedron, $F(2,32)=9.81, p<$ .001 ; tetrahedron, $F(1,16)=11.83, p<.01$; square, $F(2$, $32)=21.14, p<.001$. For the cube, planned comparisons revealed that participants were more accurate when the fourfold symmetry axis was aligned with the axis of rotation than when the twofold symmetry axis was so aligned, $F(1$, $16)=10.73, p<.01$. Performance for the threefold symmetry axis was in between the fourfold and twofold axes and was not statistically different from either one, $p>1$. For the octahedron, the fourfold symmetry axis led to superior performance over both the threefold and the twofold axes, $F(1,16)=14.22, p<.01$, and $F(1,16)=9.35, p<$ .01 , respectively. Percentage correct for the threefold and twofold axes did not differ, $p>1$. For the square, the fourfold and twofold symmetry axes were not different from each other, $F<1.0$. Both the fourfold and twofold axes were superior to the oblique orientation, $F(1,16)=26.98$, $p<.001$, and $F(1,16)=26.68, p<.001$, respectively.

Across the objects, performance always was high if the object was a generalized cone about the axis of rotation. The differences among the objects at these orientations were not statistically reliable, $F<1.0$, even though these orientations have three different amounts of symmetry about the axis of rotation. For the octahedron and tetrahedron, there was an interaction between orientation of the axis of rotation and orientation of the object to the axis: octahedron, $F(4,64)=$ $4.04, p<.01$; tetrahedron, $F(2,32)=3.70, p<.05$. In particular, performance was good either when the axis of rotation was aligned to the environment or the object was a generalized cone about the axis of rotation. 
There were no statistically reliable differences between the cube and octahedron, the two objects dual to each other. When these objects were considered together, there was an interaction between the orientation of the axis of rotation to the environment and the orientation of the object to the axis (a comparison that had not been statistically reliable for the cube considered alone), $F(4,64)=3.35, p=.01$.

The range of accuracy in this experiment was quite large. The participants were correct $95 \%$ of the time when the rotations were aligned with the environment and the objects were generalized cones about the axis of rotation. Performance dropped to $36 \%$ correct when the rotations were fully oblique to the environment and the objects rotated about their lowest symmetry axes (at which they are not generalized cones).

Response time. Mean response time, as a function of object, orientation of the axis of rotation to the environment, and orientation of the object to the axis of rotation, is presented in Figure 10. These means are for the total times per trial, from the initiation of the display to the completion of the report procedure (the second mouse click). The total time is used because participants were permitted to look back and forth from the display to the response apparatus, and often they did that. If the initial viewing times are examined separately, the same trends emerge.

The pattern of mean response time mirrored the pattern of percentage correct, $r=-.93, t(31)=13.67, p<.001$. Therefore, statistical comparisons are not reported separately. It is noteworthy, however, that the range of response times in this experiment was quite large. Mean response time was $8.9 \mathrm{~s}$ when the axis of rotation was aligned with the environment and the objects were generalized cones

Object Axis Aligned with the Axis of Rotation

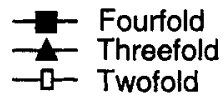

Cube

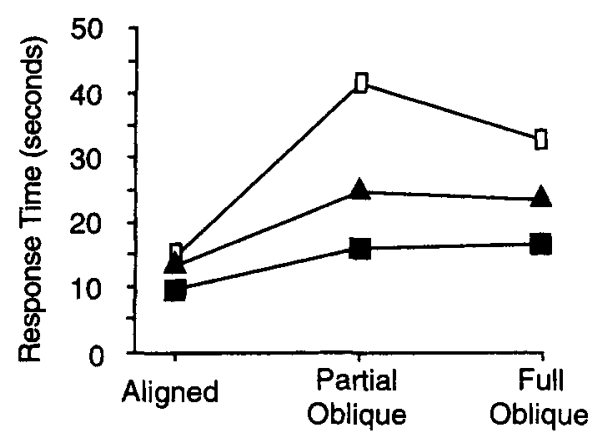

Tetrahedron

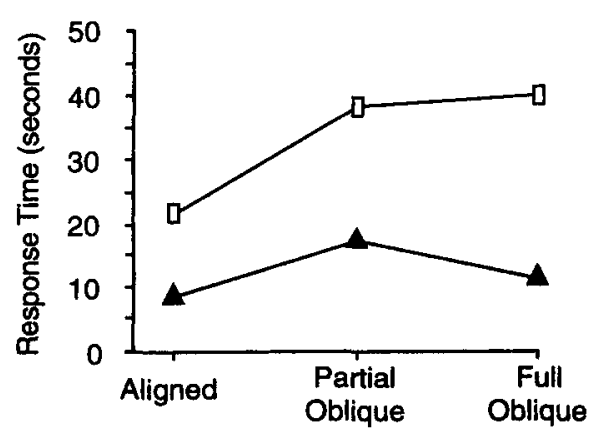

Octahedron

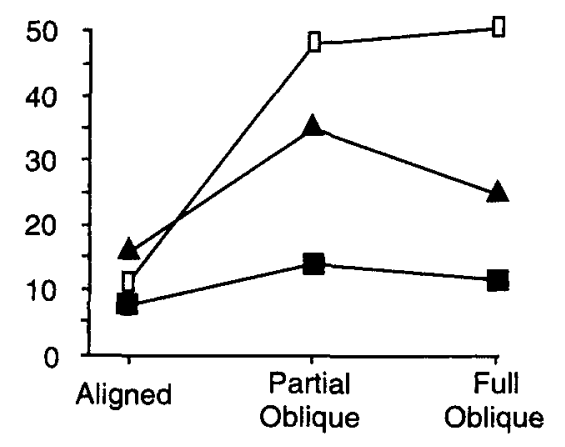

Square

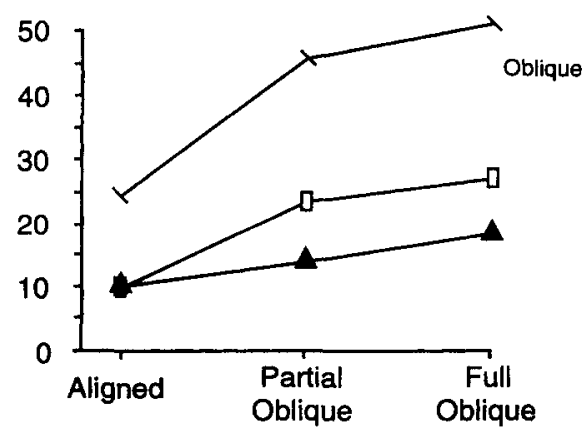

\section{Orientation of the Axis of Rotation}

Figure 10. Response time in Experiment 1 as a function of object, orientation of the axis of rotation, and orientation of the object to the axis of rotation. 
about the axis. This time included reaching for the orientation indicator, manipulating it, and placing it in the polyhedron. Mean response time rose to $43.5 \mathrm{~s}$ when the axis of rotation was fully oblique to the environment and the objects were in their least effective orientations to the axis.

\section{Discussion}

The ability to indicate the orientation of the axis and planes of a simple rotational motion ranged from relatively fast and accurate response to slow and inaccurate response. This was true even though the orientations and the response alternatives were limited to the 13 canonical directions in $35-45^{\circ}$ intervals. So long as the axis of rotation was aligned to the environment, the motions generally were perceived to be simple rotational motions. If the axis of rotation was oblique to the environment, the orientation of the object to the axis made a large difference. The results very clearly extended findings from the imagination of rotation to the perception of rotation.

Given the findings for this set of four objects, if one were to make a single suggestion of how to make rotations appear simple when the axes of rotation are oblique to the environment, that suggestion would be to orient the objects as generalized cones about the axes of rotation. The cube and octahedron rotating about their fourfold symmetry axes, the tetrahedron rotating about its threefold symmetry axis, and the square rotating about its twofold symmetry axis were readily perceived to be rotating about axes fixed in space. Below the level of symmetry at which the object was a generalized cone, participants had much more difficulty identifying the orientations of the motions. In addition, the same level of symmetry could lead to very different outcomes across different objects. This is demonstrated by perception of the rotation of two of the Platonic solids, the octahedron and tetrahedron, about their threefold symmetry axes. The tetrahedron is a generalized cone about this axis, and participants typically could identify the axis of rotation. The octahedron is not a generalized cone about this axis, and participants typically could not identify the axis. Note also that by conventional standards, the twofold symmetries of the Platonic solids yield symmetric objects. However, perception of rotations about these symmetry axes was not impressive (38\% correct after a response time of $42 \mathrm{~s}$ ). It should be noted, however, that performance was well above chance for these rotations, and the symmetry of the objects may have contributed to this.

The one object for which performance did not drop substantially when the object was not a generalized cone about the axis of rotation was the cube. When the threefold symmetry axis of the cube was aligned with the axis of rotation, performance still was relatively good. Note, however, that the cube at this orientation is a generalized cone in the top and bottom thirds of the object. Moreover, in the oblique orientations of the axis of rotation, one end of the cube was turned toward the participant so that the majority of what could be seen was a generalized cone.

\section{Experiment 2}

The results of Experiment 1 imply that alignment of the axis of a generalized cone with the axis of a simple rotation makes the rotation perceptually simple, even if the axis of rotation is oblique in the environment. However, it is important to consider the unique properties of the set of objects in that experiment. Of particular interest, the three simpler Platonic solids are generalized cones with regular polygons as cross-sections. The cross-section of the square is a straight line. Perhaps it is generalized cones with symmetric cross-sections that lead to the perception of simple rotational motions. Experiment 2 was designed to test perception of the rotation of generalized cones with a variety of irregular cross-sections. These generalized cones again had straight axes and cross-sections normal to the axes.

A second basic property of the objects used in Experiment 1 is that they all are relatively compact. The tetrahedron is the best 4-point approximation to a sphere, the octahedron is the best 6-point approximation to a sphere, and the cube is the best 8-point approximation to a sphere. In contrast, a number of authors have suggested that elongation of an object is a common source of information about the major axis of an object-relative reference system (Humphreys, 1983; Marr \& Nishihara, 1978; Palmer, 1989). In Experiment 2 , the cube, octahedron, and tetrahedron were elongated in a 2:1 ratio along their twofold symmetry axes, the axes that led to some of the worst performances in Experiment 1. The question was whether this elongation when aligned with the axis of rotation would make the rotations appear simpler.

One possible reason that rotations of the Platonic solids about their lower symmetry axes did not appear simple in Experiment 1 is that the objects contained higher symmetries. Perhaps there was a tendency to perceive the objects in terms of the higher symmetries, and this perception interfered with seeing the lower symmetries aligned with the rotation. In Experiment 2, the three simpler Platonic solids also were compressed in a 2:1 ratio along the axis of twofold symmetry. Both the elongation and the compression preserved the twofold rotation and reflection symmetry of the objects but eliminated all competing symmetries. Alignment of the twofold symmetry axes of the compressed objects with the axis of rotation was intended to give an indication of the effectiveness of symmetry on the perception of simple rotation in the absence of competing symmetry and where there was no elongation along the axis of rotation.

\section{Method}

Participants. Participants were 16 Emory University undergraduates ( 9 women and 7 men). They participated for course credit in an introductory course in psychology.

Materials. The display apparatus, viewing conditions, and type of display were the same as in Experiment 1. There were five basic types of display, as illustrated in Figure 11. First, the cube, octahedron, and tetrahedron were oriented as generalized cones to the axis of rotation. Second, the twofold symmetry axes of these 
objects were aligned with the axis of rotation. These two types were intended to provide baseline performance. The third type of display was composed of generalized cones with irregular crosssections. Eight objects were created by factorially combining three variables. The objects could have quadrilateral or trilateral crosssections, there were two versions of each of these, and the objects could be prismatic or could come to a point. The objects with quadrilateral cross-sections came to a point at both ends, forming irregular octahedra, the objects with trilateral cross-sections came to a single point, forming irregular tetrahedra. In every case, the cross-sections were made to be as irregular as possible. In particular, the angles of the quadrilaterals were in the ratio 1.5:1. The angles of the trilaterals were in the ratios $1.5: 1$ and $2.5: 1$. The surface areas and volumes of the prismatic shapes were the same as those of the cube. The surface areas and volumes of the pointed shapes were the same as those of the octahedron or the tetrahedron. The fourth set of objects was formed from elongating the Platonic solids along the twofold symmetry axes in a $2: 1$ ratio. The fifth type was formed from compressing the Platonic solids along the twofold axes, in a 1:2 ratio of height to width. The surface areas and volumes of the compressed and elongated shapes were the same as those of the corresponding Platonic solids. Across all five types of display, there were 20 oriented objects.

The axis of rotation always was oblique to the environment. There were four partially oblique orientations of the axis of rotation; the axis was in either the horizontal or the sagittal plane and was at $45^{\circ}$ to the principal axes of those planes. There also were four fully oblique orientations of the axis (these can be seen in Figure 8). Rotations could be in either direction about the axis of rotation. Thus, the complete set of displays included 16 different rotations. Each participant saw every one of the 20 oriented objects rotating with its critical object axis aligned with a partially oblique and a fully oblique axis of rotation, making 40 trials for each

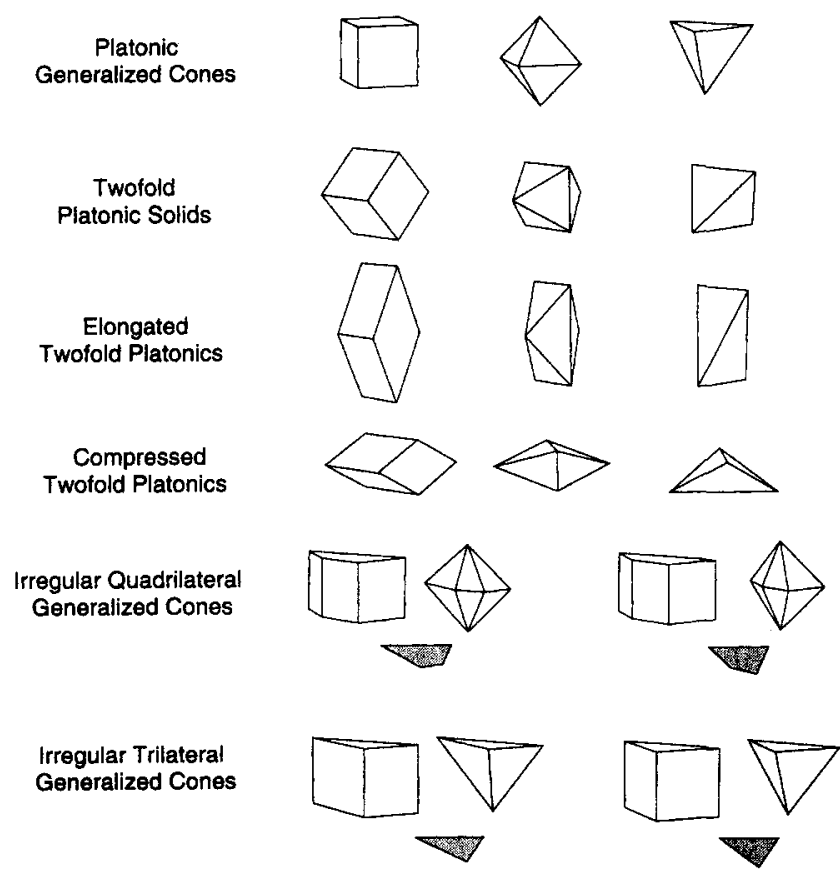

Figure 11. The objects and orientations in Experiment 2, relative to the vertical. The gray polygons illustrate the shapes of the cross-sections of the irregular generalized cones. participant. The various tetrahedral objects (including the irregular ones) were oriented to the axis of rotation with the base surface facing front one half of the time and with the opposing vertex facing front one half of the time.

Procedure. The procedure was the same as in Experiment 1 until the experimental trials. In Experiment 2, there was only one block of trials. The participant was asked to take a break after every 10 trials. In addition, there were no cardboard models of the objects shown to participants ahead of time. There were four separate lists of trials. The particular partially oblique or fully oblique axis used and the direction of rotation were varied within participant and counterbalanced across objects and participants. There were eight separate random orders of trials.

\section{Results}

Accuracy. The primary analysis was an analysis of variance with two repeated measures: type of object structure aligned with the axis of rotation and orientation of the axis of rotation. Type of object structure had five levels: Platonic generalized cone, irregular generalized cone, elongated objects, compressed objects, and twofold Platonic solids. Orientation of the axis of rotation had two levels: partially oblique and fully oblique. In preparation for the analysis, each participant's data from each of the 10 cells of this design were averaged.

The percentage of correct responses for each type of object structure at both types of orientation of the axis of rotation is presented in Figure 12. As expected, there was a large effect of the type of object structure aligned with the axis of rotation, $F(4,56)=23.79, p<.001$. The Platonic solids led to the highest and the lowest accuracy, depending on whether they were oriented as generalized cones or as twofold symmetries to the axis of rotation, $F(1,14)=$ $39.91, p<.001$. The irregular generalized cones led to accuracy much the same as the Platonic generalized cones, $F(1,14)=3.72, p=.07$. The elongated objects led to an intermediate but relatively high level of accuracy (compared with the irregular generalized cones, $F(1,14)=10.84, p<$ $.01)$. The compressed objects also led to intermediate levels

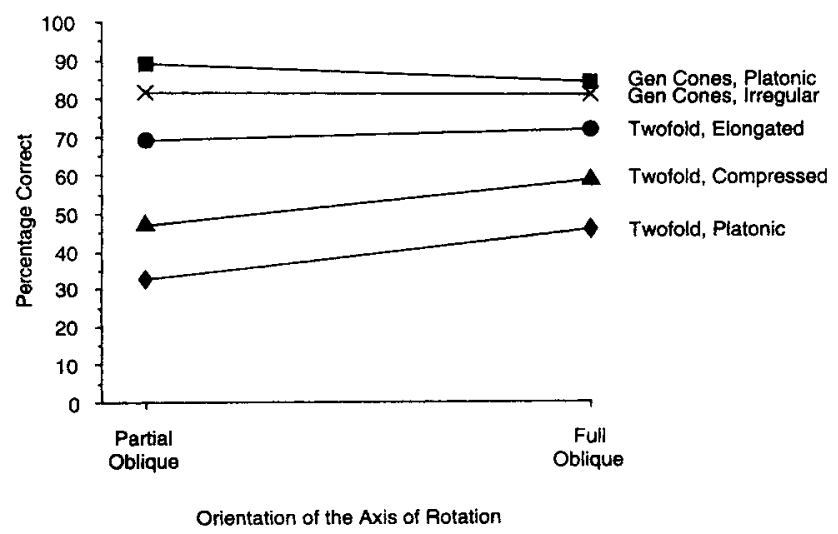

Figure 12. Percentage correct in Experiment 2 as a function of the orientation of the axis of rotation and the object structure aligned with the axis of rotation. $(\mathrm{Gen}=$ generalized). 
of accuracy but levels substantially lower than those of the elongated objects: compared with the elongated objects, $F(1,14)=8.77, p=.01$; compared with the twofold Platonic solids, $F(1,14)=7.28, p<.05$.

There were no general effects on accuracy produced by the variety of irregular generalized cones. One interaction approached statistical significance, $F(1,14)=3.75, p<$ .07 . In particular, although the objects with trilateral crosssections led to equivalent performance, objects with quadrilateral cross-sections led to better performance if they were dipyramidal rather than prismatic (for the simple effect at the quadrilaterals, $F(1,14)=4.73, p<.05)$. There was not a statistically reliable difference between partially and fully oblique orientations of the axis of rotation nor an interaction involving this variable.

Response time. Mean response time for each type of object structure at each orientation of the axis of rotation is presented in Figure 13. As in Experiment 1, the pattern of mean response times closely mirrored the pattern of percentage correct, $r=-.98, t(8)=15.62, p<.001$. Statistical comparisons therefore are not reported separately for response time.

\section{Discussion}

The ability to indicate the orientation of the axis and planes of a rotational motion again ranged from relatively fast and accurate response to slow and inaccurate response. As expected, the Platonic solids oriented as generalized cones and as twofold symmetries to the axis of rotation provided the easiest and most difficult rotations, respectively. Generalized cones with irregular cross-sections led to performance nearly equivalent to the Platonic generalized cones, although there was a slight superiority of the more regular objects. The elongated objects led to intermediate but relatively high levels of performance. This is especially interesting given that these objects were elongations of the twofold symmetries of the Platonic solids, object structures that led to poor performance. The compressed versions of

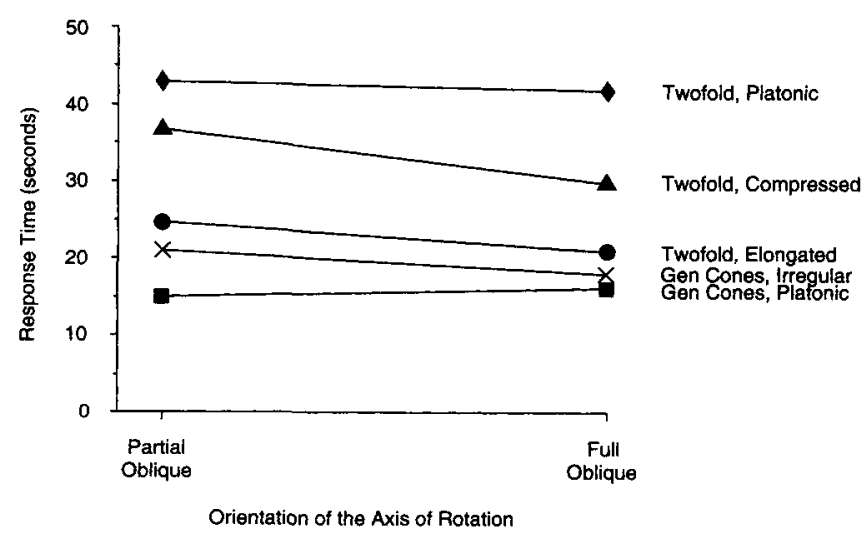

Figure 13. Response time in Experiment 2 as a function of the orientation of the axis of rotation and the object structure aligned with the axis of rotation. (Gen = generalized). these twofold symmetries also led to intermediate levels of performance, but these levels were toward the low end of the range (i.e., $53 \%$ correct after 33 s). Thus, eliminating higher symmetries in the Platonic solids improved perception of rotations about the twofold symmetry axes. However, the twofold symmetries, in the absence of elongation of the objects, still were not powerful in leading to perception of simple rotations (although, again, performance was above chance).

\section{General Discussion}

Rotation is a common and fundamental form of motion (Gibson, 1957; Shepard, 1984). Nevertheless, most people are not able to imagine every physically simple form of rotation. Even though a rotation involves only the circular motion of a square about a rod fixed in space, if the rotation is double oblique, the typical person will be unable to imagine it (e.g., Pani, 1993; Pani \& Dupree, 1994; see also Just \& Carpenter, 1985; Massironi \& Luccio, 1989; Parsons, 1987). Given such findings, the question arises as to whether the constraints on the imagination of rotation exist also in perception (see also Kaiser, Proffitt, \& Anderson, 1985; Proffitt \& Gilden, 1989). The present experiments were designed to investigate the perception of rotations of a set of basic shapes, including the square, the simpler regular polyhedra, and a variety of shapes derived from the regular polyhedra. It was demonstrated that double-oblique rotations of objects do not appear to be simple rotational motions. The rotations appear to be continuous motion of rigid objects (Green, 1961), but they do not appear to be simple rotations. It can be concluded that perceived rotation has a spatial organization that is separable from its properties as continuous motion (see also Carleton \& Shepard, 1990a, 1990b; Cutting \& Proffitt, 1982; Gilden, 1991; Pani, 1993, 1994). This organization is seen readily in certain circumstances but not in others (see also Shiffrar \& Shepard, 1991).

\section{Alignment With the Environment}

When a rotational motion is aligned with the principal axes and planes of the environment, the rotation is perceived to be simple, independent of the orientation of the object to the axis of rotation. It is important to note that in this circumstance the person need not be able to fully organize the global structure of the object relative to the motion. This is demonstrated for rotation about the vertical when the cube is oriented with opposite corners vertical, when the octahedron is oriented with opposite surfaces vertical, or the tetrahedron is oriented with opposite edges vertical (i.e., when the cube and octahedron have threefold symmetry axes vertical and the tetrahedron has a twofold axis vertical; see Figure 4). If a typical participant were to perceive a rotation about the vertical of one of these oriented objects and indicate the orientation of the motion and then were to attempt to indicate the shape of the object when it has that orientation to the vertical (e.g., by pointing to imaginary 
corners of the object), he or she would fail to do so accurately. Thus, Hinton (1979) found that people generally were unable to imagine the cube with opposite corners vertical. Pani, Zhou, and Friend (1995) had individuals view the cube rotating about the vertical for as long as they wished before stopping the display and indicating how the corners of a static cube are arranged. These participants left the displays on for an average of $35 \mathrm{~s}$, nearly three times longer than participants needed in the present Experiment 1 to indicate the axis and planes of the rotation. The imagination of the tipped cube, even after these long viewing times, was still quite inaccurate, and much more inaccurate than imagination of the other orientations of the cube.

A rotational motion forms a circularly symmetric space stretched along the axis of rotation. This kinematic space is a solid of revolution and a highly regular variant of the generalized cone (Pani, 1993, 1994; Pani \& Dupree, 1994). As a rotation progresses, it is only with respect to the axis of this kinematic space, the axis of rotation, that the features of the object maintain a constant slant. Alignment of the axis of rotation with a salient axis of the environment determines that the orientations of features of the object are the same relative to a principal axis of the environment and to the axis of rotation. Thus, perception of the orientation of the features of the object relative to the two axes is mutually reinforcing. It is then possible to see the constant slant of the features of the object to the axis of rotation and the circular motion of the features about the axis. A somewhat similar phenomenon occurs when static symmetries are made more salient by alignment with a principal axis of the environment (Goldmeier, 1972; Palmer, 1980; Palmer \& Hemenway, 1978; Pani, Zhou, \& Friend, 1995; Rock, 1983; Rock \& Leaman, 1963).

\section{Alignment With the Object}

Global properties of objects that make rotations appear simple. When objects have definite directions in space related primarily to the structures of the objects, it is reasonable to speak of object-relative reference systems (e.g., Biederman \& Gerhardstein, 1993; Corballis, 1988; Hinton, 1979; Marr \& Nishihara, 1978; McMullen \& Jolicoeur, 1992; Palmer, 1975, 1989; Rock, 1983). The question is, What properties of objects provide object-relative reference systems such that a rotational motion aligned with such a reference system is seen to be simple?

It has been suggested that the generalized cone is a volumetric primitive in the representation of object shape (Biederman, 1987, 1990; Binford, 1971; Brooks, 1981; Marr \& Nishihara, 1978). One basis for this suggestion is that the generalized cone has a definite axial structure (Marr \& Nishihara, 1978). It should be clear how to assign the major axis of an object-relative reference system to a generalized cone. Interestingly, if rotations are organized in terms of the kinematic spaces associated with axes and planes of rotation, then the geometric similarity between rotations and generalized cones is quite striking. If the generalized cone has psychological importance, rotations should appear simple when they are aligned with the axis of a generalized cone. In separate experiments, this was the case. The square, the three simpler Platonics solids, and a set of generalized cones with irregular cross-sections led to perception of simple rotational motions when the axes of the generalized cones were aligned with the axes of rotation. The Platonic solids led to slightly superior performance; the regularity of the cross-section does provide some additional benefit in the perception of the motions.

Elongation is another form of information that people could use to determine the major axis of an object-relative reference system (Humphreys, 1983; Marr \& Nishihara, 1978; Palmer, 1989). If this potential information actually is used, then rotations should appear simple when the axis and planes of motion are aligned with an axis of elongation. In Experiment 2, object structures that previously were not useful in the perception of rotation led to relatively successful perception when they were elongated in a $2: 1$ ratio and aligned with the axis of rotation. Overall, two global properties of objects that have been suggested to be useful in fixing the major axes of object-relative reference systems were effective in making a rotational motion salient when these global properties were aligned with the motion.

Rotation-reflection symmetry is a property of objects that often makes them appear well structured (e.g., Garner, 1974; Palmer, 1985, 1989; Palmer \& Hemenway, 1978; Pomerantz \& Kubovy, 1986). However, rotation and reflection symmetry did not play a strong role in the perception of rotational motion in these experiments (in contrast to the conclusions of Shiffrar \& Shepard, 1991). Most important, a single amount of symmetry could lead to very different outcomes. For example, the octahedron rotating about its threefold symmetry axis did not lead to perception of simple rotations, whereas the tetrahedron rotating about its threefold symmetry axis did. (Other relevant findings are noted in the discussions of the individual experiments.) On the other hand, performance in both experiments always was above chance, and it is quite possible that rotationreflection symmetry contributed to this. It also is true that different forms of spatial regularity can be used in combination. Thus, the Platonic generalized cones, with rotationreflection symmetry about the conic axis, were more readily seen to be simple rotations than the irregular generalized cones in Experiment 2.

Breakdown of the perception of simple rotation. We have been discussing the effects of the orientations of objects to the axes and planes of rotation on the perception of the motions, but this relationship also can influence the perceptual organization of the objects. For example, an octahedron is a generalized cone, but it is a generalized cone in three orthogonal directions. If the octahedron did not move, a given conic axis oblique in the environment would likely not be salient (Goldmeier, 1972; Palmer, 1980; Palmer \& Hemenway, 1978; Rock, 1983; Rock \& Leaman, 1963). As the octahedron rotates about a conic axis, however, only that axis has a fixed direction in space. An object axis that can be used to organize the object is made uniquely salient by its invariance across a change of orientation produced by the rotational motion. Overall, perception of 
the rotation must be a cooperative process. A rotational motion influences spatial organization of the object and is seen to be a simple rotation by its alignment with the organized structure.

An isotropic object, such as a homogeneously textured sphere, can be arbitrarily oriented to an axis of rotation, and a rotation of the sphere appears simple (see Johansson, 1950; Lappin, Doner, \& Kottas, 1980; Restle, 1979). It is not the case, then, that oblique-axis rotations must be aligned with objects that have unambiguous orientations for the rotations to be perceived as simple. Rather, a rotating object presents a succession of orientations, and the person must be able to relate these orientations to each other in terms of the structure of a simple rotation: an axis fixed in space, invariant slant of the features of the object to the axis, and circular motion of the features, with constant angular velocity, in parallel planes aligned along the axis. A homogeneously textured sphere has no intrinsic orientation, and the changing orientations of the sphere are easily seen as sets of elements that, either individually or in concentric rings, form circular motions in planes of rotation.

In other cases, the successive orientations of an object produced by a rotation cannot be perceptually organized in terms of the structure of a simple rotation. For example, consider the motion that most obviously leads to a breakdown in the perception of simple rotation, the doubleoblique rotation of the square (see Figures 9 and 10). The most obvious property of this motion, either perceptually or analytically, is that as the square spins it is sometimes aligned with the environment in one direction (e.g., in the frontal plane), sometimes fully oblique to the environment, and sometimes aligned with the environment in a second direction (e.g., horizontal; see Figure 2). The orientations of the object sampled across time constitute a variety of canonical orientations, and there is no higher organization of the object with a stable relationship to the axis and planes of rotation. In this case, the motion is perceived to be continuous but unstable change of orientation.

Radical change of the orientations of surfaces of objects also is salient in the rotation of the simpler Platonic solids about their twofold symmetry axes. And, as with doubleoblique rotations of the square, there are not salient higher organizations of the objects aligned with the axis of rotation that include the surfaces in structures with stable orientations. Note that for every one of the object structures that did not lead to perception of simple rotations, there is some feature of the object that actually is aligned with the axis of rotation. If the participant were to selectively attend to these features, performance would be much improved. For example, the threefold octahedron has a triangular surface centered on and aligned with the axis of rotation. It is the tendency to see the object as having connected surfaces and an overall organization that makes it so difficult to selectively attend to what would be useful features of the objects for identifying the nature of the motion. No doubt participants could be trained to find these isolated features, but this would say little about the nature of normal perception.

Individual properties of objects that make rotations appear simple. To explain more fully the importance of generalized cones to the perception of rotation, it would be possible to build on theories of object recognition in which generalized cones have a unique role in the representation of objects (Biederman, 1987, 1990; Marr, 1982; Marr \& Nishihara, 1978). Perceivers may be particularly sensitive to generalized cones (see Biederman, 1987, 1990). In the context of the perception of rotation, however, there is much support for considering the generalized cone to contain a type of geometric regularity and for the importance of generalized cones to be due to the importance of geometric regularity in perception (e.g., Attneave, 1954, 1981, 1982; Garner, 1974; Leeuwenberg, 1971; Leyton, 1992; Palmer, 1982, 1983; Pani, 1994; Pani, Zhou, \& Friend, 1995; Pomerantz \& Kubovy, 1986; Wertheimer, 1950).

Consider that when a square is aligned with the axis and planes of a rotational motion, the motion is perceived to be simple. It is possible to define the square in these orientations to be a generalized cone, but it is a rather minimal one. When the square is normal to the axis of rotation, it is a single cross-section and not one that has been translated along an axis. When the square is parallel to the axis of rotation, the cross-section is a line rather than the twodimensional contour generally supposed to be a constituent of the generalized cone. For rotations of the square that are perceived to be simple, it is most reasonable to appeal to the concept of alignment. The square is aligned with the axis and planes of rotation, and the axis and planes of rotation have a fixed orientation in space. Throughout the motion, orientations that are salient with respect to the square are stable with respect to the environment. The axis and the planes of motion can be related to these stable orientations of the square (also see Pani, Jeffres, et al., in press).

When the class of generalized cones is confined to those with straight axes and cross-sections normal to the axes, generalized conic polyhedra have a number of basic geometric regularities related to the conic axis (Pani, 1994; Pani, Zhou, \& Friend, 1995). First, a shape will have at least one well-defined cross-section, and such cross-sections are aligned with the axis of the cone (by definition). For prismatic solids, all edges, surfaces, and cross-sections are aligned with the axis. Where a generalized cone is not prismatic, it tends to converge to the axis (i.e., to be pyramidal, or conic in the specific sense). Related to these properties, there are strong limits on the degree to which edges and surfaces of a generalized cone can vary in orientation relative to the conic axis. First of all, the slants of edges and surfaces relative to the axis are highly constrained. The more equilateral the cross-section of the object, the more uniform the slants of edges and surfaces. In addition, the radial positions of edges and surfaces about the conic axis are the same up and down the generalized cone (i.e., one phase structure describes the positions of these features at any point along the axis). These constraints on the orientations of object features about an object axis contrast with the large shifts in orientation of object features about the axes of the Platonic solids that lead to poor perception of simple rotation (see Figures 4 and 11; Pani, 1994; Pani, Zhou, \& Friend, 1995).

Overall, the basic property of objects that makes rotations 
appear simple when that property is aligned with the axis and planes of rotation is a salient spatial organization with a clear direction. The prototypic example of such an object is a generalized cone with an equilateral cross-section elongated along the conic axis (e.g., a rocket). Such objects contain a number of specific properties, however, that most likely contribute to the perception of simple rotation. These properties include the alignment of edges and surfaces with an axis, convergence of edges and surfaces to an axis, homogeneity of surface orientations about an axis, alignment of cross-sections to an axis, and elongation along an axis.

\section{Geometric Regularity in the Structure of Objects and Motions}

We have described rotation in terms of an organized space associated with the axis of rotation. This type of symmetric space is familiar from the study of 3D form (e.g., Hilbert \& Cohn-Vossen, 1952). We have suggested that alignment of reference axes is critical to perceiving rotations, just as it is critical to perceiving static arrangements (see also Pani, Jeffres, et al., in press). Finally, we have suggested that object structures supposed to be critical to the perception of objects are critical to the perception of the rotation of objects. In all, certain forms of spatial organization are fundamental both for the perception of motion and for the perception of form (Pani \& Dupree, 1994). We wish to extend the discussion of similarities between the perception of rotation and the perception of objects. Many theorists have suggested that the concept of geometric regularity is fundamental to the explanation of the spatial organization of form (e.g., Attneave, 1954, 1981, 1982; Garner, 1974; Leeuwenberg, 1971; Leyton, 1992; Palmer, 1982, 1983; Pani, 1994; Pani, Zhou, \& Friend, 1995; Pomerantz \& Kubovy, 1986; Wertheimer, 1950). In the remaining paragraphs, we briefly point out the relevance of the concept of geometric regularity to a general conceptualization of the perception of rotational motion.

The term symmetry has many meanings (Weyl, 1952). Typically, it refers to reflection symmetry (e.g., Koffka, 1935 ) and often to rotation and reflection symmetry (e.g., Garner, 1974; Palmer \& Hemenway, 1978). But increasingly the technical usage of the term covers all types of repetition across spatial transformation (Burn, 1985; Palmer, 1983; Pani, 1994; Smart, 1988; Stewart \& Golubitsky, 1992). Some symmetries are combinations of more elementary types. For example, spiral symmetry, often called screw symmetry, is repetition across a combination of translation and rotation (Hargittai \& Pickover, 1992). In this broader usage, the generalized cone embodies a type of symmetry, either simple translational symmetry, or symmetry produced by a combination of translation and dilation (Pani, 1994). It is noteworthy in this regard that illustrations of generalized cones typically provide examples that have rotation and reflection symmetry, and that illustrations of rotation and reflection symmetry typically show generalized cones (unlike, say, the threefold octahedron).
Alignment to a reference system also is an instance of symmetry in the general sense (Pani, Jeffres, et al., in press). For example, two parallel lines or surfaces can be made congruent by a simple translation of one entity into the other. Similarly, a line normal to a reference plane forms a reflection symmetry everywhere about the line. If the reference plane is part of an orthogonal reference system (e.g., the ground plane and the vertical direction of gravity), the line normal to the plane forms an angle that is identical to the right angles already existing in the reference system. The angle formed by the line can be made congruent to an angle in the reference system by a simple translation of the line.

If one equates physical simplicity with symmetry, then the straight line and the circle are the two simplest curves (Hilbert \& Cohn-Vossen, 1952), and aligned orientations are the two simplest orientations. Given that simple rotational motion includes circular motion in parallel planes aligned along an axis, human spatial organization of rotation in terms of the axis and planes of rotation preserves the status of rotation as one of the simplest physical structures in nature. Human perceivers succeed in organizing a given rotation in this way, however, only when the rotation is geometrically simple in its relationship to other spatial reference systems related to the motion. The rotation is perceived to be physically simple only when it can be seen to be aligned with the object or the environment. When the rotation cannot be seen in this way, it appears only as continuous motion in which the orientation of the object changes. The present study, then, contributes to the evolution of the concept of pragnanz toward a description of the human sensitivity to regularity in the perceptual world.

\section{References}

Appelle, S. (1972). Perception and discrimination as a function of stimulus orientation: The oblique effect in man and animals. Psychological Bulletin, 78, 266-278.

Attneave, F. (1954). Some informational aspects of visual perception. Psychological Review, 61, 183-193.

Attneave, F. (1981). Three approaches to perceptual organization. In M. Kubovy \& J. R. Pomerantz (Eds.), Perceptual organization. Hillsdale, NJ: Erlbaum.

Attneave, F. (1982). Pragnanz and soap bubble systems: A theoretical exploration. In J. Beck (Ed.), Organization and representation in perception (pp. 11-29). Hillsdale, NJ: Erlbaum.

Biederman, I. (1987). Recognition by components: A theory of human image understanding. Psychological Review, 94, 115147.

Biederman, I. (1990). Higher-level vision. In D. N. Osherson, S. M. Kosslyn, \& J. M. Hollerback (Eds.), Visual cognition and action (pp. 41-72). Cambridge, MA: MIT Press.

Biederman, I., \& Gerhardstein, P. C. (1993). Recognizing depthrotated objects: Evidence and conditions for three-dimensional viewpoint invariance. Journal of Experimental Psychology: $\mathrm{Hu}$ man Perception and Performance, 19, 1162-1182.

Binford, T. O. (1971, December). Visual perception by computer. Paper presented at IEEE Systems Science and Cybernetics Conference, Miami, FL.

Brooks, R. A. (1981). Symbolic reasoning among 3-D models and 2-D images. Artificial Intelligence, 17, 285-348. 
Bum, R.P. (1985). Groups: A path to geometry. New York: Cambridge University Press.

Carleton, E. H., \& Shepard, R. N. (1990a). Psychologically simple motions as geodesic paths: I. Asymmetric objects. Journal of Mathematical Psychology, 34, 127-188.

Carleton, E. H., \& Shepard, R. N. (1990b). Psychologically simple motions as geodesic paths: II. Symmetric objects. Journal of Mathematical Psychology, 34, 189-228.

Corballis, M. C. (1988). Recognition of disoriented shapes. Psychological Review, 95, 115-123.

Cutting, J. E., \& Proffitt, D. R. (1982). The minimum principle and the perception of absolute, common, and relative motions. Cognitive Psychology, 14, 211-246.

Garner, W. R. (1974). The processing of information and structure. Potomac, MD: Erlbaum.

Gasson, P. C. (1983). The geometry of spatial forms. West Sussex, England: Ellis Horwood.

Gibson, J. J. (1957). Optical motions and transformations as stimuli for visual perception. Psychological Review, 63, 288-295.

Gilden, D. L. (1991). On the origins of dynamical awareness. Psychological Review, 98, 554-568.

Goldmeier, E. (1972). Similarity in visually perceived forms. Psychological Issues, 8, (1), 1-135. (Original work published 1936)

Green, B. F. (1961). Figure coherence in the kinetic depth effect. Journal of Experimental Psychology, 62, 272-282.

Hargittai, I., \& Pickover, C. A. (Eds.). (1992). Spiral Symmetry. River Edge, NJ: World Scientific.

Hecht, H., \& Proffitt, D. R. (1991). Apparent extended body motions in depth. Journal of Experimental Psychology: Human Perception and Performance, 17, 1090-1103.

Hilbert, D., \& Cohn-Vossen, S. (1952). Geometry and the imagination. New York: Chelsea.

Hinton, G. (1979). Some demonstrations of the effects of structural descriptions in mental imagery. Cognitive Science, 3, 231-250.

Holden, A. (1971). Shapes, space, and symmetry. New York: Columbia University Press.

Howard, I. P. (1982). Human visual orientation. New York: Wiley.

Humphreys, G. W. (1983). Reference frames and shape perception. Cognitive Psychology, 15, 151-196.

Just, M. A., \& Carpenter, P. A. (1985). Cognitive coordinate systems: Accounts of mental rotation and individual differences in spatial ability. Psychological Review, 92, 137-172.

Johansson, G. (1950). Configurations in event perception. Stockholm: Almqvist \& Wiksell.

Kaiser, M. K., Proffitt, D. R., \& Anderson, K. (1985). Judgments of natural and anomalous trajectories in the presence and absence of motion. Journal of Experimental Psychology: Learning, Memory, and Cognition, 11, 795-803.

Kappraff, J. (1991). Connections: The geometric bridge between art and science. New York: McGraw-Hill.

Koffka, K. (1935). Principles of gestalt psychology. New York: Harcourt, Brace, \& World.

Lappin, J. S., Doner, J. F., \& Kottas, B. L. (1980). Minimal conditions for the visual detection of structure and motion in three dimensions. Science, 209, 717-719.

Leeuwenberg, E. L. J. (1971). A perceptual coding language for visual and auditory patterns. American Journal of Psychology, 84, 307-349.

Leyton, M. (1992). Symmetry, causality, mind. Cambridge, MA: MIT Press.

Mach, E. (1959). The analysis of sensations. 5th ed. (C. M. Wil- liams \& S. Waterlow, Trans.). New York: Dover. (Original work published 1906)

Marr, D. (1982). Vision. San Francisco: Freeman.

Marr, D., \& Nishihara, H. K. (1978). Representation and recognition of the spatial organization of three-dimensional shapes. Proceedings of the Royal Society of London, Biological, 200, 269-294.

Massironi, M., \& Luccio, R. (1989). Organizational versus geometric factors in mental rotation and folding tasks. Perception, 18, 321-332.

McCloskey, M. (1983). Naive theories of motion. In D. Gentner \& A. L. Stevens (Eds.), Mental models (pp. 299-324). Hillsdale, NJ: Erlbaum.

McMullen, P. A., \& Jolicoeur, P. (1992). Reference frame and effects of orientation on finding the tops of rotated objects. Journal of Experimental Psychology: Human Perception and Performance, 18, 807-820.

Olson, D. R. (1970). Cognitive development: The child's acquisition of diagonality. New York: Academic Press.

Palmer, S. E. (1975). Visual perception and world knowledge: Notes on a model of sensory-cognitive interaction. In D. A. Norman \& D. E. Rumelhart (Eds.), Explorations in Cognition (279-307). San Francisco: Freeman.

Palmer, S. E. (1977). Hierarchical structure in perceptual representation. Cognitive Psychology, 9, 441-474.

Palmer, S. E. (1980). What makes triangles point: Local and global effects in configuration of ambiguous triangles. Cognitive Psychology, 12, 285-305.

Palmer, S. E. (1982). Symmetry, transformation, and the structure of perceptual systems. In J. Beck (Ed.), Organization and representation in perception (pp. 95-143). Hillsdale, NJ: Erlbaum.

Palmer, S. E. (1983). The psychology of perceptual organization: A transformational approach. In J. Beck, B. Hope, \& A. Rosenfeld (Eds.), Human and machine vision (pp. 269-340). New York: Academic Press.

Palmer, S. E. (1985). The role of symmetry in shape perception. In D. G. Bouwhuis, H. Buffart, \& E. L. J. Leeuwenberg (Eds.), Seeing and knowing [Special issue]. Acta Psychologica, 59, 67-90.

Palmer, S. E. (1989). Reference frames in the perception of shape and orientation. In B. E. Shepp \& S. Ballesteros (Eds.), Object perception: Structure and process (pp. 121-163). Hillsdale, NJ: Erlbaum.

Palmer, S. E., \& Hemenway, K. (1978). Orientation and symmetry: Effects of multiple, rotational, and near symmetries. Journal of Experimental Psychology: Human Perception and Performance, 4, 691-702.

Pani, J. R. (1989, November). Axis of rotation and orientation of the object in mental representation of rotation. Poster presented at the Annual Meeting of the Psychonomic Society, Atlanta, GA.

Pani, J. R. (1993). Limits on the comprehension of rotational motion: Mental imagery of rotations with oblique components. Perception, 22, 785-808.

Pani, J. R. (1994). The generalized cone in human spatial organization. In C. W. Tyler (Ed.), Theoretical issues in symmetry perception [Special issue]. Spatial Vision, 8, 491-502.

Pani, J. R., \& Dupree, D. (1994). Spatial reference systems in the comprehension of rotational motion. Perception, 23, 929-946.

Pani, J. R., Jeffres, J. A., Shippey, G. T., \& Schwartz, K. J. (in press). Imagining projective transformations: Aligned orientations in spatial organization. Cognitive Psychology.

Pani, J. R., \& Zhou, H. (1993, November). Spatial organization in the mental imagery of fundamental solids. Poster presented at 
the Annual Meeting of the Psychonomic Society, Washington, DC

Pani, J. R., Zhou, H., \& Friend, S. M. (1995). Perceiving and imagining Plato's solids: The generalized cylinder in spatial organization of $3 D$ structures. Manuscript submitted for publication.

Parsons, L. M. (1987). Visual discrimination of abstract mirrorreflected three-dimensional objects at many orientations. Perception \& Psychophysics, 42, 49-59.

Pomerantz, J. R., \& Kubovy, M. (1986). Theoretical approaches to perceptual organization: Simplicity and likelihood principles. In K. R. Boff, L. Kaufman, \& J. P. Thomas (Eds.), Handbook of perception and human performance: Vol. 2. Cognitive processes and performance (pp. 1-46). New York: Wiley.

Proffitt, D. R., \& Gilden, D. L. (1989). Understanding natural dynamics. Journal of Experimental Psychology: Human Perception and Performance, 15, 385-393.

Proffitt, D. R., Kaiser, M. K., \& Whelan, S. M. (1990). Understanding wheel dynamics. Cognitive Psychology, 22, 342-373.

Restle, F. (1979). Coding theory of the perception of motion configurations. Psychological Review, 86, 1-24.

Rock, I. (1973). Orientation and form. New York: Academic Press.

Rock, I. (1983). The logic of perception. Cambridge, MA: Bradford/MIT Press.

Rock, I., \& Leaman, R. (1963). An experimental analysis of visual symmetry. Acta Psychologica, 21, 171-183.

Shepard, R. N. (1984). Ecological constraints on internal representation: Resonant kinematics of perceiving, imagining, thinking, and dreaming. Psychological Review, 91, 417-447.

Shepard, R. N., \& Cooper, L. A. (1982). Mental images and their transformations. Cambridge, MA: MIT Press.
Shiffrar, M. M., \& Shepard, R. N. (1991). Comparison of cube rotations around axes inclined relative to the environment or to the cube. Journal of Experimental Psychology: Human Perception and Performance, 17, 44-54.

Smart, J. R. (1988). Modern geometries (3rd ed). Pacific Grove, CA: Brooks/Cole.

Stewart, I., \& Golubitsky, M. (1992). Fearful symmetry: Is God a geometer? Cambridge, MA: Blackwell.

Todd, J. T. (1982). Visual information about rigid and nonrigid motion: A geometric analysis. Journal of Experimental Psychology: Human Perception and Performance, 8, 238-252.

Treisman, A., \& Gormican, S. (1988). Feature analysis in early vision: Evidence from search asymmetries. Psychological Review, 95, 15-48.

Tversky, B. (1981). Distortions in memory for maps. Cognitive Psychology, 13, 407-433.

Ullman, S. (1979). The interpretation of structure from motion. Proceedings of the Royal Society of London, Biological, 203, 405-426.

Wallach, H., \& O'Connell, D. N. (1953). The kinetic depth effect. Journal of Experimental Psychology, 45, 205-217.

Wertheimer, M. (1950). Laws of organization in perceptual forms. In W. D. Ellis (Ed.), A source book of Gestalt Psychology (pp. 71-88). New York: Humanities Press. (Abridged version of a paper originally published in German in 1923).

Weyl, H. (1952). Symmetry. Princeton, NJ: Princeton University Press.

Received February 23, 1994

Revision received September 23, 1994

Accepted November 28, 1994 